\title{
At a glance: Proteomics in China
}

\author{
HE FuChu \\ State Key Laboratory of Proteomics, Beijing Proteome Research Center, Beijing Institute of Radiation Medicine, Beijing 102206, China
}

Received December 12, 2010

Citation: He F C. At a glance: Proteomics in China. Sci China Life Sci, 2011, 54: 1-2, doi: 10.1007/s11427-010-4131-0

Proteomics is a new science that focuses on the comprehensive analysis of proteins in intact organisms or in molecule machineries, organelles, cells, tissues, or organs. It has become an important area of interests in life sciences and has propelled the rapid development of cutting-edge biotechnology in the 21 st century. In response to this, the Human Proteome Organization (HUPO) was launched in 2001. The mission of HUPO is to advocate and promote proteomics worldwide and to initiate the Human Proteome Project (HPP) to decode the human genome and to establish the proteomic basis of human physiology and pathology. Eleven projects including the Human Liver Proteome Project (HLPP) led by China are under way. Governments, multinational companies, particularly pharmaceutical and analytical instrument companies, as well as the genomic company Celera Genomics, have invested heavily, hoping to seize the huge potential of proteomics.

Proteomics was introduced in China in 1997, three years after the first use of the term. With the logistical support of the Chinese central and local governments, China's National Medium and Long Term Science and Technology Development Plan (2006-2020) has made proteome research a core part of the first Major Scientific Research Program. The China Human Proteome Organization (CNHUPO) was founded in 2003. Since its inception, CNHUPO has played an important role in encouraging the rapid development of proteomics in China through collaboration, training and education by supporting scientific projects, annual conferences and international exchanges. Excitingly, the Sixth Annual Conference of the CNHUPO in 2009 attracted nearly 600 participants, a dramatic 3 -fold increase since the first conference in 2003.

Proteomics has emerged as a major research area in China through the supports of governments and scientists. Many pro-

email: hefc@nic.bmi.ac.cn teome research laboratories, facilities and centers have been established in recent years, including the Research Center for Proteome Analysis (Key Lab of Proteomics, Institute of Biochemistry and Cell Biology, Shanghai Institutes of Biological Sciences, Chinese Academy of Sciences, Shanghai, 200031, China), the Institutes of Biomedical Sciences (Fudan University, Shanghai, 200032, China), Proteomics Key Lab (Institute of Basic Medicine, Chinese Academy of Medical Sciences), and the Beijing Proteome Research Center (BPRC).

Founded in 2002, the BPRC was the first proteomics institute established in China. Now it has become a top proteomics institute. At the Center, proteomics is used as a means to exploring and understanding the complex physiological and pathological processes taking place in the liver and other organs/tissues/cells. BPRC relocated to the Zhongguancun Life Science Park on 29 October 2005 and started its rapid development. Now, BPRC is the executive headquarters of HLPP. The Ministry of Science and Technology (MOST) of China approved BPRC as the core unit of the State Key Laboratory of Proteomics (Proteome-SKY) in 2009.

The BPRC has led multiple national and international proteomics projects. The Chinese Human Liver Proteome Project (CNHLPP) is one of them. CNHLPP is an ambitious project that aims to construct a human liver proteome reference map by cataloguing protein expression, modification and protein-protein interaction in the liver. The reference map will serve as a starting point for the systems biology view of liver physiology and pathology and lay the foundation for understanding and eventually curing the liver diseases.

The largest dataset of a human organ proteome, the first version of the human liver proteome (HLP), has been established by the Chinese Human Liver Proteome Profiling Consortium. This dataset comprises of 6788 proteins each with at least two peptides that are identified at $5 \%$ false discovery rate. 
Concurrently, they obtained the human liver transcriptome (HLT) dataset using the same sample, which consists of 11205 transcripts. They found direct association between HLT and HLP. Preliminary analysis of HLP revealed that proteins involved in liver-specific functions, such as bile transport, bile acid synthesis, and bilirubin metabolism, are well represented as expected; proteins involved in metabolism, nutrient transport, and blood coagulation, as well as complement systems comprise the majority of HLP. MAP kinases and signaling components in calcium, adhesion, insulin, and adipocytokine actions are identified. Half of the HLP are discovered in liver for the first time, and $82.5 \%$ of them are of low abundance. For example, four members of the cytochrome P450 family and three ion channels are described for the first time in liver. The overlap in coagulation, anticoagulation, fibrinolysis, and complement system between human liver and plasma proteomes is particularly noteworthy, demonstrating a significant positive correlation between the abundance of coagulator proteins in liver and plasma. The success of the human liver proteome project will have a high impact on the understanding of liver diseases such as non-alcoholic steatohepatitis (or NASH), HBV infection, and hepatic cancers. The dataset of human liver proteome has built a solid foundation for future identification of new biomarkers and targets of therapeutic intervention in liver diseases.

The Center has also developed a protein-protein interaction map and has comprehensively compared the protein expression patterns of human fetal liver samples from different development stages with intriguingly different physiological characteristics. This dataset represents the first comprehensive description of the human liver protein interaction network. They utilized the high-throughput yeast two-hybrid technology to map the interactions of 5026 human liver proteins. A network of 3484 interactions amongst 2582 proteins was established and $72 \%$ of them were validated with three independent assays.
The biological significance of this interaction network has been illustrated by the topological analyses of metabolic enzymes, liver-specific and liver-disease proteins, as well as their associated proteins. Of those analyzed, 166 proteins are expected to cause a liver phenotype if their function is disrupted and 58 proteins are potential liver-disease candidates. This dataset will help to obtain a system biology outlook of protein interactions in human liver cancer cells. Such information will transform the field of basic biology research in liver function and impact all biological research where protein interactions play a regulatory role.

To facilitate the use of the valuable resources and make them accessible to the wider scientific community, we have made available publicly databases such as dbLEP (http:// dblep.hupo.org.cn), liverbase (http://liverbase.hupo.org.cn) and livermap (http://livermap.hupo.org.cn). The Center has accumulated a large volume of primary biological information and has begun to appreciate the complexicity of the liver proteome.

To provide unparalleled capacity for proteomics in the next five to ten years, they are building a large national scientific facility for proteomics, dubbed Pilot Hub of ENcyclopedical proteomIX (PHOENIX). The PHOENIX project is the first specialized facility for life sciences of its kind among Chinese national large scientific facilities and is headed by BPRC. It will host most of the large modern instruments for protein science with mass spectrometry, optical molecular imaging, cryo-electron microscopy (Cryo-EM), and nuclear magnetic resonance (NMR). If such progress continues, it will not be long before we find 'proteomics' appearing regularly in the language of popular science.

I hope this special issue-At a glance: Proteomics in China will stimulate your interest in Chinese proteomics. Enjoy the special issue that includes eight papers representing a broader overview of proteomics in China.

Thank you very much.

\section{Biographical Sketch}

He Fuchu, $\mathrm{PhD}$, is a Member of the Chinese Academy of Sciences, a Member of the Academy of Sciences for the Developing World, and is currently the Director of the State Key Laboratory of Proteomics. He is the President of the Beijing Proteome Research Center and a Professor at the Beijing Institute of Radiation Medicine. He Fuchu is a council member of the Human Proteome Organization (HUPO), co-chair (inaugural chair) of the HUPO Human Liver Proteome Project (HLPP), the vice-president of AOHUPO, and the president of CNHUPO. He received his B.S. degree in genetics from Fudan University, Shanghai, in 1982 and earned his M.S. degree in biochemistry and his $\mathrm{PhD}$ in cell biology from the Beijing Institute of Radiation Medicine. His major fields of research are proteomics, genomics, bioinformatics and systems biology, with a special interest in liver physiology and pathology. He is a senior editor of Proteomics and Proteomics-Clinical Application and is an editorial board member of Molecular \& Cellular Proteomics and the Journal of Proteome Research and an executive editor of the Journal of Proteomics \& Bioinformatics. He has published more than 200 papers in international peer-reviewed journals, including Science, Nature Cell Biology, Nature Genetics, Nature Biotechnology, Nature Methods, Nature Protocols, PNAS, EMBO J, Gastroenterology, Hepatology, Genome Res, and Mol Cell Proteomics.

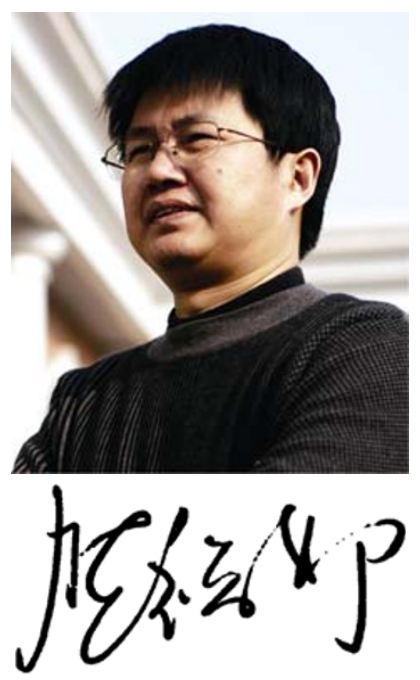

Open Access This article is distributed under the terms of the Creative Commons Attribution License which permits any use, distribution, and reproduction in any medium, provided the original author(s) and source are credited. 